\title{
Preschoolers' novel noun extensions: shape in spite of knowing better
}

\section{Henrik Saalbach* and Lennart Schalk}

Institute of Behavioral Sciences, ETH Zurich, Zurich, Switzerland

\section{Edited by:}

Haley Vlach, University of California Los Angeles, USA

\section{Reviewed by:}

Lynn K. Perry, University of lowa, USA

Daniel Yurovsky, Indiana University, USA

\section{*Correspondence:}

Henrik Saalbach, Institut f.

Verhaltenswissenschaften,

Eidgenössische Technishe Hochschule

Zürich, UNO C 14, Universitätsstrasse

41, 8092 Zürich, Switzerland.

e-mail: henrik.saalbach@ifv.gess.

ethz.ch
We examined the puzzling research findings that when extending novel nouns, preschoolers rely on shape similarity (rather than categorical relations) while in other task contexts (e.g., property induction) they rely on categorical relations. Taking into account research on children's word learning, categorization, and inductive inference we assume that preschoolers have both a shape-based and a category-based word extension strategy available and can switch between these two depending on which information is easily available. To this end, we tested preschoolers on two versions of a novel-noun label extension task. First, we paralleled the standard extension task commonly used by previous research. In this case, as expected, preschoolers predominantly selected same-shape items. Second, we supported preschoolers' retrieval of item-related information from memory by asking them simple questions about each item prior to the label extension task. Here, they switched to a category-based strategy, thus, predominantly selecting same-category items. Finally, we revealed that this shape-to-category shift is specific to the word learning context as we did not find it in a non-lexical classification task. These findings support our assumption that preschoolers' decision about word extension change in accordance with the availability of information (from task context or by memory retrieval). We conclude by suggesting that preschoolers' noun extensions can be conceptualized within the framework of heuristic decision-making. This provides an ecologically plausible processing account with respect to which information is selected and how this information is integrated to act as a guideline for decision-making when novel words have to be generalized.

Keywords: word learning, categorization, preschoolers, shape bias, decision-making

\section{INTRODUCTION}

Preschool-aged children tend to generalize novel nouns for objects on the basis of perceptual similarity rather than (non-obvious) categorical relations (e.g., Clark, 1973; Bowerman, 1978; Gentner, 1978; Landau et al., 1988). This so-called shape bias has been found to be particularly strong when category relation and shape similarity are separated, and pitted against each other, as in the forced-choice match-to-sample paradigm used by a number of developmental studies with English-speaking children (e.g., Baldwin, 1992; Imai et al., 1994; Golinkoff et al., 1995) as well as Chinese- and German-speaking children (e.g., Imai et al., 2010). In the forced-choice match-to-sample paradigm, children are told to extend the name of an object (the standard) to one of two or more other objects. Imai et al. (1994), for example, found that 3-yearolds and 5-year-olds who had been taught a novel name for the standard (e.g., cake) preferred to extend the name to shape-similar but categorically unrelated objects (e.g., hat) rather than to objects that belonged to the same category (e.g., pie) or were thematically related (e.g., birthday gift). Adults, in contrast to preschoolers, predominantly relied on categorical relations. Preschoolers' strong reliance on shape in label extension tasks is inconsistent with findings from studies on preschoolers' inductive inferences. Here, when tested on similar or even the same material, children did not rely on shape similarity but, just like adults, recruited non-obvious categorical relations (e.g., Gelman and Markman, 1986; Saalbach and Imai, 2006).

The present work attempts to resolve this inconsistency. For this purpose, we first review two main theoretical directions on word extension, the "attention to shape" and the "taxonomic assumption" account. Then we will argue that these theories come up against specific difficulties in explaining the shape bias with respect to preschool-aged children. Finally, we propose a new account to conceptualize preschoolers' noun extension. Accordingly, preschoolers can actually switch between two strategies in novel noun extension, a shape-based and a category-based, depending on which one is prompted by the task. The present studies were conducted to evaluate our proposal.

\section{MAIN THEORETICAL DIRECTIONS ON LABEL EXTENSION}

Generally speaking there are two main theoretical approaches to account for young children's shape bias in learning novel nouns. The first approach holds that the shape bias in word learning contexts is acquired by attentional learning in the course of early (count) noun acquisition (Smith et al., 1996; Colunga and Smith, 2005, 2008). According to this account, toddlers learn a system of statistical regularities that establish a link between linguistic devices, the properties of objects, and perceptual category organization. For example, in the context of categorizing non-solid 
substances children learn to attend to material (e.g., Samuelson and Horst, 2007), whereas shape is the relevant property for categorizing solid objects, and, thus, paying attention to shape is a successful strategy for early acquisition of names for solid objects. In fact, computational approaches which modeled the acquisition of the shape bias (e.g., connectionist networks, Colunga and Smith, 2005; hierarchical Bayesian models, Kemp et al., 2007) support the assumption that relying on shape is a successful strategy that boosts early learning of solid object names. More importantly, it has been shown that supporting toddlers by directing their attention to shape indeed resulted in rapid vocabulary growth (e.g., Smith et al., 2002; Gershkoff-Stowe and Smith, 2004).

Since most of these studies have been conducted with toddlers (i.e., children aged one to three) it is not clear why preschoolers (i.e., children above 3 years of age) still rely on shape. Preschoolers have already acquired a vocabulary which also contains many names for superordinate categories (e.g., animal, food, fruit, toy, etc.) that refer to classes of objects which do not necessarily resemble each other in shape (e.g., Waxman and Gelman, 1986). This suggests that preschoolers can also rely on relations that go beyond perceptual similarities when extending novel names for objects. Yet, they still show a strong shape bias in typical word learning tasks. How can this be explained?

In contrast to the "attention to shape-account," some researchers have argued that even very young children implicitly know that the meanings of nouns are organized around categorical relations (e.g., Markman and Hutchinson, 1984; Waxman and Gelman, 1986; Markman, 1989; Waxman and Kosowski, 1990; Booth and Waxman, 2002; Booth et al., 2005; Cimpian and Markman, 2005). The so-called "taxonomic assumption" is said to help young children to narrow down the space of potential referents for a new noun. But, then, why do preschoolers favor perceptual relations over categorical relations in typical word learning tasks?

Exponents of the "taxonomic assumption" suggested two possible explanations: First, children's reliance on shape in the commonly used noun extension tasks is seen as a compensation for their incomplete knowledge about the categorical structure of the word's referent: "children often seem to think of 'like kind' as 'perceptually like kinds,' especially 'shape-similar kinds"' (Genther and Namy, 1999, p. 489). Second, the absence of categorybased noun extensions could be considered an artifact of the specific method of these studies. In fact, Cimpian and Markman (2005) argued that preschoolers may not be able to identify and reason about superordinate categories in these studies because the stimuli were presented in an unnatural (drawings instead of photos or real objects), decontextualized, and simple-shaped way. According to Cimpian and Markman children usually follow the taxonomic assumption in noun extension. In common noun extension tasks, however, they either misinterpret the novel name as a term for a specific shape rather than for a category, or they mistake the "unnatural," simple-shaped, or decontextualized shape-similar items as items of the same kind as the standards. In fact, when Cimpian and Markman used more complex or contextualized (and hence less similar) objects as experimental items in the noun extension task, they found a significant decrease of shape choices.

\section{LIMITATIONS IN EXPLAINING PRESCHOOLERS' SHAPE BIAS IN LABEL EXTENSION}

However, other findings in the literature question these explanations of preschoolers' preference for shape in label extension tasks. First, attributing the shape bias to preschoolers' incomplete conceptual knowledge about the objects to be labeled is inconsistent with research on the development of inductive reasoning. Findings indicate that preschoolers do have insight into the structure of object categories and are indeed able to recruit this knowledge when asked to project non-obvious object properties, regardless of whether or not these objects are similar in shape (e.g., Carey, 1985; Gelman and Markman, 1986, 1987; Gelman et al., 1986; Gelman and Wellman, 1991; Heyman and Gelman, 2000a,b; Imai et al., 2010). Carey (1985), for example, reported that 4-year-olds agreed that monkeys can breathe, eat, and have babies, but denied that a mechanical monkey could do the same. In other words, in spite of the perceptual similarity preschoolers did not project properties of living things onto non-living things.

Second, attributing the shape bias to a methodological artifact is questioned by findings from Imai et al. (2010; see also Saalbach and Imai, 2006). Here, using the same material, a property induction and a label extension task were carried out within a forced-choice match-to-sample paradigm. Preschoolers were presented with a standard (e.g., banana) and three choice alternatives. The choice alternatives were designed to represent three kinds of relations in each set: the shape match that was outside the standard's category but perceptually resembled the standard (feather); the category match, that belonged to the target category but was perceptually distinct from the standard (grape); and the thematic match that was thematically related to the standard (monkey). In the property induction task (Experiment 3 ), children were told that the standard had an internal property unknown to them (e.g., "This has enzyme inside") and were asked to point to the alternative which also had this property inside. Imai and colleagues found that preschoolers' pattern of inductive inference did not differ from that of adults since they clearly preferred the category match to the shape match. In the label extension task (Experiment 2), a novel count noun was used to label the standard (e.g., "This is a FEP") and the child was asked to extend it to one of the three alternative items (e.g., "Which one of these is also called FEP"). Here, Imai and colleagues found that preschoolers strongly preferred the shape match to the category match. This suggests that shape preference cannot be regarded as a pure artifact of the specific experimental material used. In spite of their ability to recruit categorical knowledge in the property induction task, preschoolers predominantly relied on shape similarity in the context of a word learning task (Deák and Bauer, 1996).

To sum up, even though research about the emergence of the shape bias has been very carefully designed and carried out, their assumptions come up against specific difficulties in explaining the shape bias with respect to preschool-aged children. In the present research, we thus examined why preschoolers show a strong shape bias in commonly used word learning tasks but are able to identify and recruit non-obvious categorical relations in other tasks and contexts. 


\section{PROMPTING SHAPE-BASED AND CATEGORY-BASED LABEL EXTENSIONS}

For this purpose it is important to note that reliance on shape similarity and reliance on categorical relations are not necessarily mutually exclusive constraints, or biases, in preschoolers' label extensions. Instead, they may just reflect different strategies which preschoolers may use alternatively, depending on the context.

Indeed, Golinkoff et al. (1995) showed that 4-year-olds shifted from a shape-based strategy to a category-based strategy when mere reliance on shape similarity was not sufficient for their decision. In their label extension task, these preschoolers were asked to extend the novel label either to an object which was similar in shape but categorically unrelated to the standard, or to an object which was similar in shape and categorically related to the standard (or to a thematically related object). Golinkoff et al. (1995) observed that 4-year-olds clearly preferred the objects which were also categorically related to the standard. Thus, when there were two shape-similar alternatives, shape similarity was insufficient as a basis for making an appropriate decision. This suggests that if a shape-based strategy is not useful for extending the noun label, preschoolers switch to the category-based strategy.

Furthermore, Gentner and Namy (1999; see also Namy and Gentner, 2002) found that when the number of standard items in a label extension task was increased, this also affected preschoolers' choice of information to rely on. In particular, if the standards were labeled with the same novel noun, providing two or more categorical related standards resulted in a shape-to-category shift, in contrast to a version with only one labeled standard. These findings indicate that preschoolers have different strategies for noun extension available and can flexibly shift between them.

We therefore assumed that the shape preference in preschoolers' novel noun extension might be due to the structure of the task. In particular, the salience of the shape similarity between standard item and shape match in typical label extension tasks might have prompted the shape-based strategy although preschoolers had also acquired, and commonly applied, a category-based strategy.

This account is partly in accordance with Cimpian and Markman (2005). As discussed above, they have argued that the shape preference in preschoolers' label extensions was a methodological artifact since stimuli items were presented in unnatural ways. On the other hand, however, Imai et al. (2010) have shown that when presented with the same kind of stimuli in a property induction task, preschoolers are able to recruit categorical information from them. This is actually a puzzling finding: Given that categorical relations were relevant in both tasks, why would salient shape similarity prevent children from recruiting categorical information only in the label extension task but not in the property induction task? In other words: Why were preschoolers able to infer properties on the basis of categorical relations whereas they relied on perceptual similarities when extending nouns? One explanation might be that relying on shape may be the most successful strategy for noun extension (but not for property induction). However, there may also be another answer to this question.

As the stimuli were the same in both tasks it stands to reason that the instruction of the induction task prompted preschoolers to rely on categorical information while the label extension instruction did not. In Imai et al. (2010), preschoolers were repeatedly asked the same question, each time with a different novel property name. For example: "This (the standard) has FEP inside. Which of them (the choice items) has also FEP inside?" Here, the critical word may be "inside." Indeed, developmental research on inductive reasoning has shown that young children understand that internal properties have a privileged status for category identity and categorical-typical functioning especially with regard to natural categories (e.g., Gelman and Markman, 1986; Gelman and Wellman, 1991; Gelman, 2003; Bulloch and Opfer, 2009). For example, in a classic study, Gelman and Markman (1986) found that 4-yearolds were more likely to make category-based inferences about internal properties (e.g., internal organs) than about properties that were determined by their perceptual appearance (e.g., weight). Thus, asking them to generalize an "inside" property may have actually triggered their conceptual knowledge. In other words, while the instruction of the property induction task conducted by Imai et al. (2010) may have helped children not to be distracted by the salient shape similarity, they had no such support in the label extension task. Here, the salient shape similarity may instead have prompted a shape-based strategy. If this holds true, we expect to find a shift from a shape-based to a category-based strategy in preschoolers' noun extensions after prompting a category-based strategy. This prompting could be done by reminding children of what they already know about the objects, for example, by asking simple questions prior to the label extension task.

This approach allows us to evaluate our own explanation of the shape bias as well as those proposed by others in the literature. If triggering conceptual information results in a shape-to-category strategy shift, this would not only confirm our own predictions (i.e., the category-based strategy being used as a result of triggering) but would also allow us to conclude that preschoolers have the relevant categorical knowledge and are capable of recruiting this knowledge for noun extension. The shape preference in typical label extension tasks may then be attributed to the salience of the perceptual similarity which triggers the shape-based strategy. However, children would not shift from a shape to a categorybased strategy after a very brief conceptual triggering if they do commonly rely on a shape-based strategy that has been learned as a successful strategy for extending nouns for solid objects. Also, no shape-to-category shift should occur if preschoolers misinterpreted the novel word as referring to (perceptual) properties of the object rather than to the object itself (for example, if FEP, as a novel label for banana, could be interpreted as meaning "long item," preschoolers would be more likely to extend the label to feather than to grapes).

\section{THE PRESENT STUDY}

In the present study, we examined whether a category-based strategy could be prompted for preschoolers' extension of novel nouns. In other words, we tested whether preschoolers shifted from a shape-based strategy to a category-based strategy after their conceptual knowledge about the objects had been triggered. For this purpose, we paralleled previous research on word and category learning by using a match-to-sample forced-choice paradigm which consisted of one standard object and three choice objects, each sharing one of the major conceptual relations - category, 

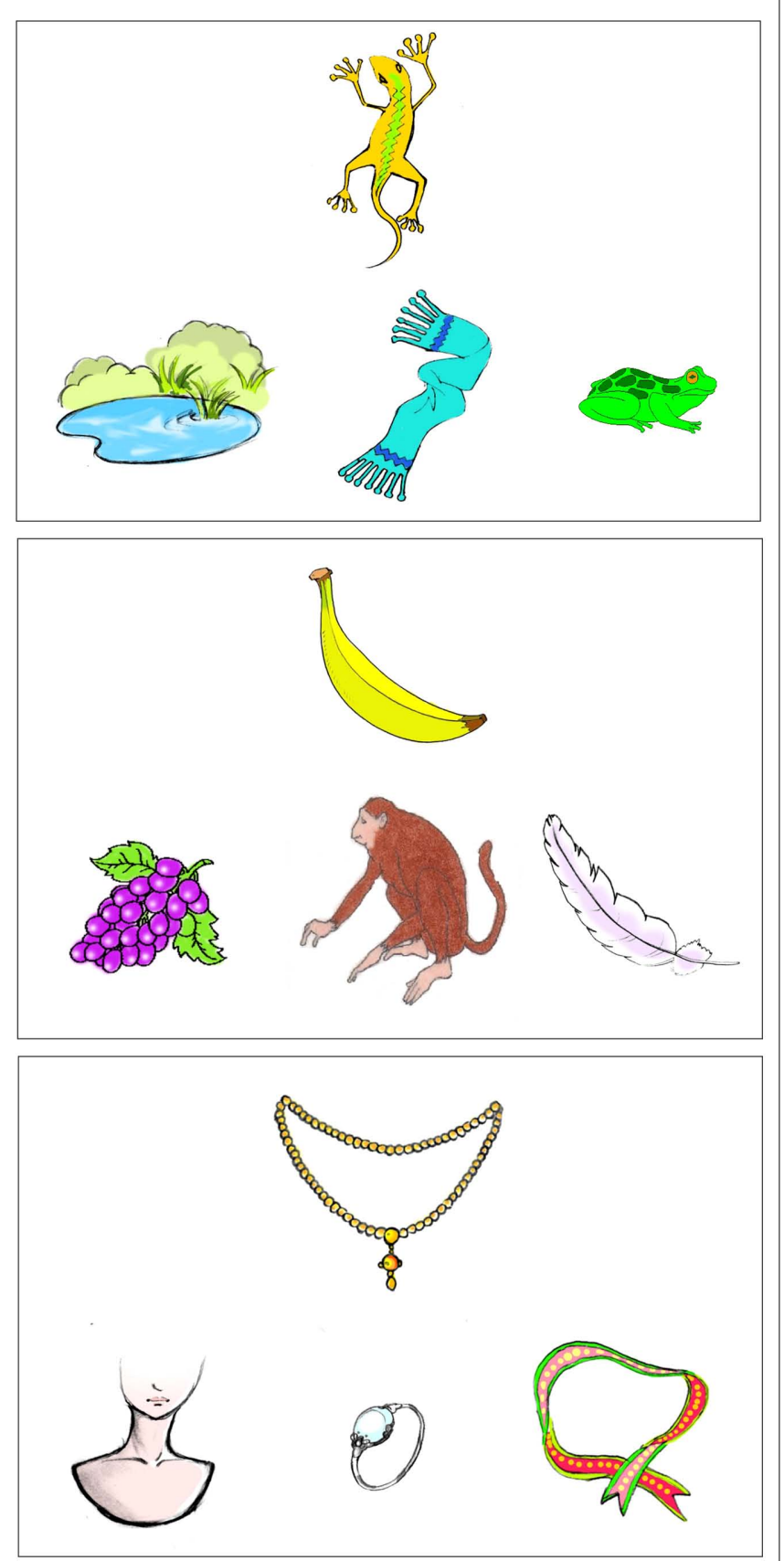

FIGURE 1 | Stimulus examples of one animal set, one plant set, and one artifact set; each with standard, category match, shape match, and thematic match.

shape, and thematic similarity - with the standard (see Figure 1 for examples of the stimulus sets).

In Experiment 1, we tested two versions of a label extension task. The first version was the standard label extension task which has been commonly used in word learning research: Preschoolers were asked to generalize a novel label which had been assigned to the standard. Here, we expected to replicate previous findings on preschoolers' strong reliance on shape similarity for extending novel labels. This task was also given to adults. The second version included one critical variation: In a triggering phase prior to the label extension task, preschoolers were asked questions about certain attributes of each object in the set [e.g., "Which of these can climb trees?", "Which of these keeps birds warm?", "Which of these can be eaten?" for the set with a banana (standard), grapes (category match), a feather (shape match), and a monkey (thematic match)]. The questions were constructed to trigger preschoolers' knowledge about each object and, at the same time, to be easy for them to answer. We predicted a significant shape-to-category shift in preschoolers' label extension from the standard to the triggering version.

Importantly, we did not change the stimulus materials across conditions but supported the retrieval of information only by asking simple questions. We did not provide any feedback. Answers relied solely on participants' prior knowledge. Asking these questions can thus in no way be considered to be a learning intervention which may have changed preschoolers' level of knowledge about the choice objects.

Experiments 2 and 3 were carried out to examine an alternative interpretation of the potential shape-to-category shift in preschoolers' label extension from the standard to the triggering condition. In particular, we tested whether the knowledge shift can be attributed to a procedural artifact rather than to a strategy shift after knowledge triggering.

\section{EXPERIMENT 1: LABEL EXTENSION AND KNOWLEDGE TRIGGERING}

Experiment 1 included two conditions. In the first condition, the standard condition, we conducted a standard version of the label extension paradigm. Thus, we tested which of the three relations category, shape, or thematic relation - was used by preschoolers and adults, respectively, in order to extend novel object labels. In the second condition, the triggering condition, preschoolers answered knowledge triggering questions prior to the label extension task. Thus, Experiment 1 allowed us to examine whether the pattern of preschoolers' label extensions changed after conceptual knowledge about the object had been triggered.

\section{METHOD}

\section{Participants}

Thirty-nine German-speaking preschoolers (mean age: 3:8, ranging from 3:1 to 4:3; 20 girls and 19 boys) participated in this study. Preschoolers were recruited at preschools in middle-class metropolitan areas in Zurich and randomly assigned to the conditions ( 10 girls and 10 boys to the standard condition; 10 girls and 9 boys to the triggering condition). Additionally, we tested 11 (six female and five male) undergraduates from Berlin Technical University on the standard version of the label extension task. Adult participants were paid for their participation. Participants' demographic backgrounds were the same for all of the studies reported in this paper. None of the participants in this and in the following experiments participated in more than one experiment of the present research.

\section{Materials}

The same materials were used as in Imai et al.' (2010) study; that is, 12 sets of color drawings of familiar objects. Each set showed 
four objects: the standard and three choice alternatives with different relations to the standard - a category match, a shape match, and a thematic match. Two objects were considered to be categorically related when they belonged to the same taxonomic category and shared common behavioral, physical, and internal properties. The shape relation was defined as the perceptual shape similarity between the standard and the choice alternative. The thematic relation was defined as an external relation; in other words, two objects were thematically related when they either appeared in the same setting or event or had a causal relation. The 12 quadruplets were constructed to represent three conceptual domains: animals, plants, and artifacts (see Table 1). The stimulus sets were presented on laminated cards (see Figure $\mathbf{1}$ for examples for each conceptual domain).

Materials were pilot-tested and validated in a pre-study by Imai et al. (2010). In the pilot study adult participants from Germany and China rated category and shape similarity between the standard and the three choice alternatives for each set. Category ratings were significantly higher for object pairs chosen as categorically related than for shape-similar and thematically related objects. Furthermore, shape similarity ratings were significantly higher for shape-similar object pairs than for pairs chosen as categorically and thematically related. Additional pretests ensured that children of the relevant age groups were familiar with all the objects.

For the triggering condition, questions were constructed to support preschoolers' retrieval of conceptual knowledge about the objects shown on the cards. There were three questions per set: One of the questions addressed the categorical membership of the category match and the standard, the other two addressed idiosyncratic properties of the shape and the thematic matches. In the case of the banana set, for example, preschoolers were asked the following questions: "Which of these can climb trees?" ("monkey") for the thematic match, "Which of these keeps birds warm?" ("feather") for the shape match, and "Which of these can be eaten?" ("banana" and "grape") for the standard match and the category match, respectively (see Table 2 for all questions constructed for

Table 1 | Materials of Experiment 1, 2, and 3.

\begin{tabular}{lllll}
\hline Set & Standard & Category & Shape & Thematic \\
\hline ANIMAL & & & & \\
1 & Snake & Turtle & Jump rope & Glass cage \\
2 & Eel & Guppy & Belt & Water tank \\
3 & Salamander & Frog & Scarf & Pond \\
4 & Beaver & Cat & Tie & Logs \\
PLANT & & & & \\
5 & Banana & Grape & Feather & Monkey \\
6 & Apple & Cucumber & Ball & Knife \\
7 & Carrot & Tomato & Match & Rabbit \\
8 & Onions & Peppers & Candle & Frying pan \\
ARTIFACT & Tat & Turban & Tent & \\
9 & CD & Tape & Pizza & Head \\
10 & Necklace & Ring & Ribbon & Neck \\
11 & Comb & Brush & Knife & Hair \\
12 & & &
\end{tabular}

Table 2 | Knowledge triggering questions used in Experiment 1 and 2.

\begin{tabular}{ll}
\hline Standard Question & $\begin{array}{l}\text { Correct } \\
\text { response }\end{array}$
\end{tabular}

\section{ANIMAL}

Snake

C: which of these are alive?

Turtle, Snake

S: which of these can you play with?

Jump rope

T: which of these can be used to lock

Glass cage

Eel

animals?

C: which of these are alive?

Guppy, Eel

S: which of these tightens trousers?

Belt

T: which of these can be used to keep

Water tank fish?

Salamander C: which of these are alive?

Frog,

Salamander

S: which of these protects from the

Scarf

cold?

T: which of these is where fish live?

Pond

Beaver

$\mathrm{C}$ : which of these are alive?

S: which of these can be worn around

the neck?

T: which of these can be used to lock

Cat, Beaver

Tie

animals?

\begin{tabular}{|c|c|c|}
\hline \multicolumn{3}{|l|}{ PLANT } \\
\hline \multirow[t]{3}{*}{ Banana } & C: which of these can be eaten? & Grape, Banana \\
\hline & S: which of these keeps birds warm? & Feather \\
\hline & T: which of these claims on trees? & Monkey \\
\hline \multirow[t]{3}{*}{ Apple } & C: which of these can be eaten? & $\begin{array}{l}\text { Cucumber, } \\
\text { Apple }\end{array}$ \\
\hline & $\begin{array}{l}\text { S: which of these do children like to play } \\
\text { with? }\end{array}$ & Ball \\
\hline & $\begin{array}{l}\text { T: which of these can hurt someone } \\
\text { badly? }\end{array}$ & Knife \\
\hline \multirow[t]{3}{*}{ Carrot } & C: which of these can be eaten? & Tomato, Carrot \\
\hline & $\begin{array}{l}\text { S: which of these can be used to make } \\
\text { fire? }\end{array}$ & Match \\
\hline & T: which of these likes to jump around? & Rabbit \\
\hline \multirow[t]{3}{*}{ Onion } & C: which of these is can be eaten? & $\begin{array}{l}\text { Peppers, } \\
\text { Onion }\end{array}$ \\
\hline & S: which of these lightens rooms? & Candle \\
\hline & T: which of these can be used to cook? & Frying pan \\
\hline \multicolumn{3}{|c|}{ ARTIFACT } \\
\hline \multirow[t]{3}{*}{ Hat } & $\begin{array}{l}\text { C: which of these do people wear on } \\
\text { their heads? }\end{array}$ & Turban, Hat \\
\hline & S: which of these can be used to live in? & Tent \\
\hline & T: which of these is a body part? & Head \\
\hline \multirow[t]{3}{*}{$C D$} & $\begin{array}{l}\text { C: which of these can you put in } \\
\text { somewhere to listen to music? }\end{array}$ & Tape, CD \\
\hline & S: which of these can be eaten? & Pizza \\
\hline & $\begin{array}{l}\text { T: which of these can be used to make } \\
\text { loud music? }\end{array}$ & Stereo \\
\hline \multirow[t]{3}{*}{ Necklace } & $\begin{array}{l}\text { C: which of these girls are wearing to } \\
\text { look nice? }\end{array}$ & Ring, Necklace \\
\hline & $\begin{array}{l}\text { S: which of these can be used to wrap a } \\
\text { present? }\end{array}$ & Ribbon \\
\hline & T: which of these is a body part? & Neck \\
\hline
\end{tabular}


Table 2 | Continued

\begin{tabular}{lll}
\hline Standard & Question & $\begin{array}{l}\text { Correct } \\
\text { response }\end{array}$ \\
\hline Comb & C: which of these can be used to fix hair? & Brush, Comb \\
& S: which of these can hurt someone badly? & Knife \\
& T: which of these grow on the head? & Hair
\end{tabular}

For each of the 12 sets three questions were constructed, the category question (C) required subjects to choose two pictures of one set (the standard and the category match), while the shape (S) and the thematic (T) question required them to choose one object only (the shape match and the thematic match, respectively).

Experiment 1). Two of the questions required one answer each (shape match and thematic match), whereas one of the questions required pointing to two pictures (the category match and the standard).

\section{Procedure}

Preschoolers were tested individually in a quiet room in their preschool. Testing took between 10 and $15 \mathrm{~min}$. The preschoolers were constantly praised and encouraged, but no explicit feedback was given. These procedural criteria were the same for all the following experiments.

Before the testing, preschoolers of both conditions were introduced to a monkey puppet called Jojo. They were told that the monkey wanted to learn "monkey language," and were asked to help the monkey.

In the standard condition, they were then shown one card and given time to look at all objects carefully. The experimenter pointed to all objects and told the child to look at all of them. This exploration phase had been included as the time allotted to preschoolers to explore the stimuli had been shown to influence their decision in the categorization tasks; more specifically, very short exploration times (i.e., less than $10 \mathrm{~s}$ ) elicited more shape choices (Kemler Nelson et al., 2000). After the child had explored an object set the experimenter assigned a novel label to the standard. Twelve different labels that were novel for preschoolers as well as for adults were constructed by combining vowels and consonants in an unfamiliar but phonologically possible way. The experimenter then asked the child to extend this label to one of the three choice alternatives presented on the card [e.g., "Look! This is a FEP in monkey language (pointing to the standard). Can you tell Jojo which one of these (pointing to the three choice alternatives) is also a FEP?"]. Preschoolers answered by pointing to the pictures or by giving the name of the stimulus.

In the triggering condition, preschoolers were asked questions prior to the label extension task. Within each set, the order of questions was randomized. The time preschoolers spent on one card was roughly equal in both conditions. Preschoolers enjoyed being asked the questions and had no difficulties at all in giving adequate replies (by pointing to objects or by naming objects). After the questions, preschoolers were given the label extension task for each set. Procedures for the label extension task were exactly the same as in the standard label extension condition.

Adults were tested individually in a university laboratory and were given the following instruction: "Imagine that you are
Table 3 | Mean proportion of each choice type and SD for Experiment 1,2 , and 3 .

\begin{tabular}{|c|c|c|c|c|}
\hline & \multirow[t]{2}{*}{$N$} & \multicolumn{3}{|c|}{ Choice alternatives } \\
\hline & & Category & Shape & Thematic \\
\hline \multicolumn{5}{|c|}{ Experiment 1 - Standard label extension } \\
\hline Adults & 11 & $78.0 \%{ }^{* *}(16.4)$ & $16.7 \%{ }^{* *}(16.5)$ & $5.3 \%{ }^{* *}(8.6)$ \\
\hline Preschoolers & 20 & $30.4 \%(16.5)$ & $51.7 \%^{* *}(21.7)$ & $17.9 \%^{* *}(16.1)$ \\
\hline \multicolumn{5}{|c|}{ Experiment 1 - Label extension with knowledge triggering } \\
\hline Preschoolers & 19 & $64.0 \%{ }^{* *}(25.8)$ & $25.4 \%(26.1)$ & $10.6 \%{ }^{* *}(9.6)$ \\
\hline \multicolumn{5}{|c|}{ Experiment 2 - Standard non-lexical categorization } \\
\hline Preschoolers & 15 & $36.1 \%(21.5)$ & $37.2 \%(21.6)$ & $26.7 \%(18.4)$ \\
\hline \multicolumn{5}{|c|}{ Experiment 2 - Non-lexical categorization with knowledge triggering } \\
\hline Preschoolers & 20 & $38.3 \%(22.8)$ & $30.0 \%(21.7)$ & $31.7 \%(23.0)$ \\
\hline \multicolumn{5}{|c|}{$\begin{array}{l}\text { Experiment } 3 \text { - Label extension with equally weighted knowledge } \\
\text { triggering }\end{array}$} \\
\hline Preschoolers & 17 & $52.0^{* *}(18.3)$ & $33.8(18.3)$ & $14.2^{* *}(15.2)$ \\
\hline
\end{tabular}

T-tests have been conducted to test whether the rate of a particular choice is significantly different from chance level.

** Denotes significantly different from chance level, $p<0.01$ (based on Bonferroni adjusted probabilities).

learning a new language you do not know. In that language, this (pointing to the standard) is a FEP. Which of these (pointing to the three alternatives) can also be a FEP in that language?"

\section{RESULTS}

Mean proportions and SDs of category, shape, and thematic choices for preschoolers and adults are shown in Table 3 (along with data from Experiments 2 and 3). As expected, extension of novel labels based on shape similarity was most frequent among preschoolers in the standard task. The proportion of choices of the shape match (51.7\%) and the thematic match (17.9\%) differed significantly from chance (33\%), $d=0.84$ and $d=0.96$, respectively, while the proportion of choices of the category match did not $(30.4 \%)$. In contrast to the preschoolers, adults showed a clear preference for category matches $(78.0 \%, d=2.73)$. Thus, these findings are consistent with previous research using the standard label extension task.

In the triggering condition, however, the response pattern changed significantly. Here, preschoolers predominantly chose the category match when extending the novel noun $(64.0 \%, d=1.19)$. An ANOVA comparing the percentages of category choices of the two preschool groups (standard condition and triggering condition) and the adult group revealed a significant group effect $[F(2$, $47)=24.91, p<0.01]$. Bonferroni adjusted post hoc tests revealed that preschoolers in the knowledge triggering condition and adults made more category choices than preschoolers in the standard label extension condition $(p<0.01, d=1.64 ; p<0.01, d=3.00$, respectively). There was no significant difference in the percentages of category choices between preschoolers in the triggering condition and adults $(p=0.241)$.

In order to conduct a stronger test of our prediction that preschoolers change strategies for extending novel labels after conceptual knowledge has been triggered, we classified preschoolers 
as Category-Dominant, Shape-Dominant, or Thematic/NonDominant. They were classified as Dominant when they chose the same match type on more than seven (out of 12) sets. Seven was the first integer above chance level by the binominal criterion, $p<0.05$. Participants not meeting this criterion were classified as Non-Dominant. Participants showing a Thematic-Dominant response pattern and participants showing a Non-Dominant response pattern were collapsed into one category. There were two reasons for this: First, our research question focused on the pattern of choices of the shape match and the category match, and, second, allocation of the thematic-dominant category was minimal (three preschoolers in all the experiments of the present research). The Response Dominance pattern for this Experiment is shown in Table 4 (along with the results of Experiments 2 and 3 ).

We first conducted an overall Pearson $\chi^{2}$-test across the three Dominance categories. In order to test our hypothesis more specifically, a subsequent $\chi^{2}$-test was conducted comparing only the proportions of Shape-Dominant and Category-Dominant participants. A Fisher's exact test was additionally conducted when a presumption for Pearson's $\chi^{2}$-test was violated (more than $20 \%$ of cells with expected frequencies less than five). However, we will report only results of the Pearson's $\chi^{2}$-test, as results of the Fisher's exact test showed the same pattern.

First, we compared Response Dominance Types of preschoolers in both conditions. We found a highly significant association between conditions (standard condition vs. triggering condition) and Response Dominance Type (Category-, Shape, Thematic/Non-Dominant), $\chi^{2}(2)=14.83, p<0.01$. The preplanned analysis of the proportions of Category-Dominant and Shape-Dominant preschoolers in both tasks also revealed a highly significant association, $\chi^{2}(1)=8.16, p<0.01$; Odds Ratio $=32.36$, suggesting a significant shape-to-category strategy shift in preschoolers' label extension due to knowledge triggering. While in the standard label extension task, preschoolers showed a strong preference for the shape match when extending a novel noun, they predominantly relied on categorical relations after having been asked simple questions about the objects.

Table 4 | Numbers and percentages of participants falling into the three Response Dominance categories for Experiment 1, 2, and 3.

\begin{tabular}{|c|c|c|c|c|}
\hline & $N$ & Category & Shape & Non/thematic \\
\hline \multicolumn{5}{|c|}{ Experiment 1 - Standard label extension } \\
\hline Adults & 11 & $10(90.9 \%)$ & $0(0.0 \%)$ & $1(9.1 \%)$ \\
\hline Preschoolers & 20 & $1(5.0 \%)$ & $8(40.0 \%)$ & $11(5$ \\
\hline \multicolumn{5}{|c|}{ Experiment 1 - Label extension with knowledge triggering } \\
\hline Preschoolers & 19 & $12(63.2 \%)$ & $3(15.8 \%)$ & $4(21.1 \%)$ \\
\hline \multicolumn{5}{|c|}{ Experiment 2 - Standard non-lexical categorization } \\
\hline Preschoolers & 15 & $2(13.3 \%)$ & $2(13.3 \%)$ & $11(73.3 \%)$ \\
\hline \multicolumn{5}{|c|}{ Experiment 2 - Non-lexical categorization with knowledge triggering } \\
\hline Preschoolers & 20 & $6(30.0 \%)$ & $2(1.0 \%)$ & $12(60.0 \%)$ \\
\hline \multicolumn{5}{|c|}{$\begin{array}{l}\text { Experiment } 3 \text { - Label extension with equally weighted knowledge } \\
\text { triggering }\end{array}$} \\
\hline Preschoolers & 17 & $8(47.0 \%)$ & $3(18.0) \%$ & $6(35.0 \%)$ \\
\hline
\end{tabular}

Second, we compared the Response Dominance Types between preschoolers and adults. We included only Category-Dominant and Shape-Dominant preschoolers and adults in these analyses. As expected, we found a highly significant association between Age and Response Dominance Type, $\chi^{2}(1)=11.92 ; p<0.01$; Odds Ratio $=52.39$ when comparing preschoolers in the standard label extension task to adults. This indicated that there were significantly more Category-Dominant adults than preschoolers and significantly more Shape-Dominant preschoolers than adults. In contrast, after answering the knowledge triggering questions, preschoolers' Response Dominance pattern did no longer statistically differ from the pattern of the adults, $\chi^{2}(2)=1.551$, $p=0.460$.

\section{DISCUSSION}

Taken together, the results of Experiment 1 were in line with our predictions. Triggering preschoolers' conceptual knowledge about experimental items induced a shift from a shape-based to a category-based strategy in preschoolers' novel noun extension: Asking simple questions about the experimental objects prior to the extension task resulted in more category-based choices for label extensions as compared to the standard label extension task.

However, there may be an objection to our interpretation of this striking shape-to-category strategy shift: The difference between the two conditions of Experiment 1 may have resulted from an artifact produced by the knowledge triggering procedure. Having being asked the question addressing the categorical relation between the two items, preschoolers pointed to both the standard item and the category match. In contrast, for answering the other two questions, children only needed to point to one object each (i.e., to either the shape or to the thematic match). Thus, the experimental procedure (i.e., pointing to two items vs. pointing to one item) may have produced a salience of the category match as compared to the other two choice alternatives. Preschoolers' preference for the category match in the label extension task may, then, have been due to a procedural artifact rather than a strategy shift after knowledge triggering.

\section{EXPERIMENT 2: NON-LEXICAL CATEGORIZATION}

A second experiment was thus conducted in order to test whether the shape-to-category strategy shift in preschoolers' label extensions might be due to a procedural artifact rather than to a shift to a category-based strategy induced by triggering their conceptual knowledge. To this end, we used the same materials and provided the same knowledge triggering procedure as in Experiment 1 , but administered a non-lexical categorization task instead of the label extension task. Preschoolers were asked to select the choice alternative that best "matched" the standard.

Non-lexical categorizations do not constrain a particular kind of knowledge, as two objects can "match" in many different ways including category, perceptual, and thematic relations (Saalbach and Imai, 2006; Imai et al., 2010). Thus, different information can be an appropriate basis for deciding which object to choose. We therefore expected preschoolers' choices of the category match to be influenced by knowledge triggering in a label extension task but not in a non-lexical categorization task. If knowledge triggering led to similar changes in response patterns as those observed in 
label extension, the effect in Experiment 1 could be attributed to an artifact produced by the experimental procedure. In Experiment 2, we thus tested preschoolers' non-lexical categorizations with prior knowledge triggering (the triggering condition) and without prior knowledge triggering (the standard condition).

\section{METHOD}

\section{Participants}

Thirty-five preschoolers were randomly assigned to the two conditions of Experiment 2: non-lexical categorization with and without prior knowledge triggering (mean age: 3:7, ranging from 3:1 to 4:3, 18 girls and 17 boys).

\section{Materials and procedure}

The same materials as in the previous experiment were used. The procedure also paralleled Experiment 1, but the categorization context was changed from word learning to a non-lexical categorization task. Again, preschoolers were introduced to the monkey puppet Jojo and were asked to help him in a difficult task. For each of the 12 stimuli cards, they were asked to point to the choice alternative that best matched the standard. ["Look at this (Experimenter points to the standard)! Which of these (Experimenter points to the choice alternatives) goes best with that?"]. In the triggering condition, preschoolers had to answer the same questions as in Experiment 1 (see Table 2) before they did the categorization task.

\section{RESULTS AND DISCUSSION}

In contrast to Experiment 1, the distribution of preschoolers' choices among category, shape, and thematic matches was almost equal (see Table 3 ). In both conditions, none of the three types of match choices significantly differed from chance (all $p s>0.50$ ). No differences in the percentages of choices of the category match between both conditions were found $[t(33)=0.185, p=0.86]$.

We again classified participants by response dominance type (Category-, Shape-, Thematic/Non-Dominant) as we had done in Experiment 1 (see Table 4), and submitted the dominance patterns of both conditions to a $\chi^{2}$-test. As expected, there was no significant difference between the patterns, $\chi^{2}(2)=1.36, p>0.5$.

Taken together, results of Experiment 2 showed that answering the knowledge triggering questions did not affect preschoolers' non-lexical categorization. Distribution of preschoolers' choices among the three different relation types was almost equal, regardless of whether or not they had to reply to questions prior to the categorization task. Thus, these findings suggest that the shape-tocategory strategy shift in Experiment 1 cannot be attributed to an accentuation of category matches by the experimental procedure.

\section{EXPERIMENT 3: LABEL EXTENSION WITH EQUALLY WEIGHTED KNOWLEDGE TRIGGERING}

Although, in Experiment 2, we found that the shape-to-category shift after knowledge triggering was not a methodological artifact, it is not clear to what extent the shift depended on the fact that categorical relations had been specifically triggered. It should be noted that of the three questions for each item set in Experiment 1, one was constructed to address the categorical membership of the category match and the standard while the other two addressed idiosyncratic properties of the shape and the thematic matches [e.g., "Which of these can be eaten?", "Which of these keeps birds warm?" "Which of these can climb trees?", for the set with a banana (standard), grapes (category match), a feather (shape match), and a monkey (thematic match)]. In other words, the categorical membership was the only relation between the standard item and the choice items which was explicitly addressed by the triggering questions. Thus, it was important to test whether preschoolers were able to select the relevant information even though other relations (i.e., shape similarity, thematic relation) had also been addressed by the questions. This would allow us to examine the nature of prompting a category-based strategy in label extension more closely.

To do this we again used a label extension task but changed the procedure of knowledge triggering. That is, we constructed new questions to equally address all three kinds of relations (category, shape, and thematic). For example, for the set with a banana (standard), grapes (category match), a feather (shape match), and a monkey (thematic match) we asked the preschoolers the following questions: "Which of these (pointing to the choice items) can be eaten like this (pointing to the standard)?", "Which of these (pointing to the choice items) looks like this (pointing to the standard)?", "Which of these (pointing to the choice items) likes to eat this (pointing to the standard)?"

\section{METHOD \\ Participants}

Seventeen preschoolers participated in this experiment (mean age: 3:7, ranging from 3:2 to 3:11, nine girls and eight boys).

\section{Materials and procedure}

Materials and procedure paralleled the knowledge triggering condition of Experiment 1. Only the knowledge triggering questions that were asked prior to the label extension task were changed. Each of the three questions addressed one of the three relations between the standard and the choice items. The experimenter first pointed to all the choice objects and the standard and then asked the three triggering questions that equally highlighted the different relations between each of the choice items and the standard (see Table 5 for all questions used in Experiment 3). As in Experiment 1 , preschoolers enjoyed the task and had no difficulties in giving adequate replies. After the child had replied to all questions the experimenter asked him or her to extend the novel labels, following the procedure of Experiment 1.

\section{RESULTS AND DISCUSSION}

Preschoolers predominantly extended the novel noun to the category match (see Table 3 for mean percentages and SDs of choices of category, shape, and thematic matches). The proportion of category choices $(52 \%)$ differed significantly from chance $(d=1.02)$ while the proportion of shape choices did not. The proportion of category choices in Experiment 3 was significantly higher than that of participants in the standard label extension condition of Experiment $1[t(35)=3.94, p<0.01, d=1.29]$ whereas it did not significantly differ from the proportion of category choices in the triggering condition of Experiment $1[t(34)=1.683$, $p>0.1]$.

As in the previous experiments, preschoolers were classified according to their response dominance (see Table 4). This 
Table 5 | Equally weighted knowledge triggering questions used in Experiment 3.

\begin{tabular}{|c|c|c|}
\hline Standard & Question & $\begin{array}{l}\text { Correct } \\
\text { response }\end{array}$ \\
\hline \multicolumn{3}{|l|}{ ANIMAL } \\
\hline Snake & $\begin{array}{l}\text { C: which of these is alive like this? } \\
\text { S: which of these looks like this? } \\
\text { T: which of these can be used to lock this up? }\end{array}$ & $\begin{array}{l}\text { Turtle } \\
\text { Jump rope } \\
\text { Glass cage }\end{array}$ \\
\hline Eel & $\begin{array}{l}\text { C: which of these is alive like this? } \\
\text { S: which of these looks like this? } \\
\text { T: which of these can be used to lock this up? }\end{array}$ & $\begin{array}{l}\text { Guppy } \\
\text { Belt } \\
\text { Water tank }\end{array}$ \\
\hline Salamander & $\begin{array}{l}\text { C: which of these is alive like this? } \\
\text { S: which of these looks like this? } \\
\text { T: which of these is where this lives in? }\end{array}$ & $\begin{array}{l}\text { Frog } \\
\text { Scarf } \\
\text { Pond }\end{array}$ \\
\hline Beaver & $\begin{array}{l}\text { C: which of these is alive like this? } \\
\text { S: which of these looks like this? } \\
\text { T: which of these can be used to lock this up? }\end{array}$ & $\begin{array}{l}\text { Cat } \\
\text { Tie } \\
\text { Cage }\end{array}$ \\
\hline \multicolumn{3}{|l|}{ PLANT } \\
\hline Banana & $\begin{array}{l}\text { C: which of these can be eaten like this? } \\
\text { S: which of these looks like this? } \\
\text { T: which of these likes to eat this? }\end{array}$ & $\begin{array}{l}\text { Grape } \\
\text { Feather } \\
\text { Monkey }\end{array}$ \\
\hline Apple & $\begin{array}{l}\text { C: which of these can be eaten like this? } \\
\text { S: which of these looks like this? } \\
\text { T: which of these can be used to cut this? }\end{array}$ & $\begin{array}{l}\text { Cucumber } \\
\text { Ball } \\
\text { Knife }\end{array}$ \\
\hline Carrot & $\begin{array}{l}\text { C: which of these can be eaten like this? } \\
\text { S: which of these looks like this? } \\
\text { T: which of these likes to eat this? }\end{array}$ & $\begin{array}{l}\text { Tomato } \\
\text { Match } \\
\text { Rabbit }\end{array}$ \\
\hline Onion & $\begin{array}{l}\text { C: which of these can be eaten like this? } \\
\text { S: which of these looks like this? } \\
\text { T: which of these can be used to cook this? }\end{array}$ & $\begin{array}{l}\text { Peppers } \\
\text { Candle } \\
\text { Frying pan }\end{array}$ \\
\hline \multicolumn{3}{|l|}{ ARTIFACT } \\
\hline \multirow[t]{2}{*}{ Hat } & $\begin{array}{l}\text { C: which of these keeps the head warm like } \\
\text { this? }\end{array}$ & Turban \\
\hline & $\begin{array}{l}\text { S: which of these looks like this? } \\
\text { T: where to put this? }\end{array}$ & $\begin{array}{l}\text { Tent } \\
\text { Head }\end{array}$ \\
\hline \multirow[t]{3}{*}{$C D$} & $\begin{array}{l}\text { C: which of these can you put in somewhere } \\
\text { to listen to music like this? }\end{array}$ & Tape \\
\hline & S: which of these looks like this? & Pizza \\
\hline & T: which of these can be used to hear this loud? & Stereo \\
\hline \multirow[t]{3}{*}{ Necklace } & $\begin{array}{l}\text { C: which of these are girls wearing to look nice } \\
\text { like this? }\end{array}$ & Ring \\
\hline & S: which of these looks like this? & Ribbon \\
\hline & T: where to put this around? & Neck \\
\hline \multirow[t]{3}{*}{ Comb } & $\begin{array}{l}\text { C: which of these can be used to fix hair like } \\
\text { this? }\end{array}$ & Brush \\
\hline & S: which of these looks like this? & Knife \\
\hline & T: which of these can you tighten with this? & Hair \\
\hline
\end{tabular}

For each of the 12 sets three questions were constructed, one addressing the category relation $(C)$, one addressing the shape relation (S), and one addressing the thematic relation $(T)$ between the standard and the choice objects.

dominance pattern was again compared to the dominance pattern of the standard label extension condition in Experiment 1. The overall $\chi^{2}$-test revealed a significant association between condition (standard condition of Experiment 1 and equally weighted knowledge triggering of Experiment 3) and Response Dominance (Category-, Shape-, and Non-Dominant), $\chi^{2}(2)=9.00$, $p=0.011$. The pre-planned contrast comparing only the numbers of participants classified as Category- and Shape-Dominant across Experiments 1 and 3 also revealed a significant effect, $\chi^{2}(1)=5.31, p=0.021$, Odds Ratio $=21.3$, indicating a strong shape-to-category strategy shift in the standard label extension condition from Experiment 1 to Experiment 3.

We additionally compared the dominance pattern of Experiment 3 to the dominance pattern of the triggering condition of Experiment 1 . Both the overall $\chi^{2}$-test and the specific contrast of the numbers of participants classified as Category- and ShapeDominant revealed no significant effects $\left[\chi^{2}(2)=1.01, p=0.579\right.$; $\chi^{2}(1)=0.19, p=0.664$, respectively].

In sum, Experiment 3 revealed that in order to prompt the category-based strategy in preschoolers' label extension, no specific triggering of their knowledge about categorical relations was needed. The findings rather suggest that it is sufficient to trigger conceptual knowledge in general, including knowledge about relations other than category membership (perceptual similarity, thematic relations). Preschoolers are thus capable of selecting the relevant information in order to appropriately extend the label. We will come back to this issue in the General Discussion.

\section{GENERAL DISCUSSION}

Our research started out from the puzzling finding that preschoolers preferred to rely on shape similarity for extending novel nouns in a commonly used noun extension context but predominantly relied on categorical relations in a property induction context. We assumed that preschoolers have a shape-based and a category-based strategy available and can flexibly shift between them according to which strategy is prompted by the task context. If this was true, we expected to find a shape-to-category shift in preschoolers' novel noun extensions after a shift to the categorybased strategy had been induced by triggering their conceptual knowledge about the objects to be labeled. The three experiments presented in this research supported our hypothesis.

In Experiment 1, we first replicated a strong reliance on a shape-based strategy when preschoolers were tested on a typical label extension task. However, when preschoolers answered simple questions about the objects prior to the label extension task, they predominantly extended the novel label to the category match. In the two remaining experiments, we demonstrated that the shapeto-category strategy shift must not be seen as an artifact produced by the specific experimental procedure of Experiment 1 .

In Experiment 2, we paralleled the design of Experiment 1 but used a non-lexical categorization task instead of a label extension task. In contrast to Experiment 1, preschoolers' response patterns did not change as a result of triggering: There was no preferred conceptual relation regardless of whether or not questions were asked before the categorization task. In Experiment 3, we again used a label extension task but introduced an equally strong triggering of preschoolers' knowledge about all three kinds of conceptual relations between the standard and the choice objects (i.e., shape match, taxonomic match, and thematic match). In spite of the different procedure, the result pattern was similar to that of the knowledge triggering condition of Experiment 1. 
The results of our research are consistent with previous findings reported in the literature. First, we replicated the strong shape bias in preschoolers' novel label extensions by using simple and decontextualized stimuli (e.g., Baldwin, 1992; Imai et al., 1994; Cimpian and Markman, 2005; Saalbach and Imai, 2006). Second, we confirmed that preschoolers have two strategies available for extending novel nouns: a shape-based strategy and a categorybased strategy, and that they are able to switch between them (e.g., Golinkoff et al., 1995; Gentner and Namy, 1999).

More importantly, our findings go beyond previous research as they suggest that preschoolers' strong shape preferences in typical word learning tasks can be attributed to the specific characteristics of the task. In particular, the salient shape similarity may prompt them to use the shape-based strategy. However, when in label extension tasks the use of the category-based strategy was induced by triggering their knowledge about the objects, preschoolers were found to rely on categorical relations for extending novel nouns to (almost) the same extent as adults did. This finding allows us to reject other potential explanations of the shape bias. First, preschoolers' shape bias cannot be attributed to a lack of conceptual knowledge about the objects, given that we did not provide any new information but only triggered preschoolers' existing knowledge. Furthermore, we found that preschoolers can flexibly shift away from the shape strategy although this strategy has been very efficient for generalizing words for solid objects. Finally, preschoolers seem not to be guided by the assumption that novel words they are taught in a typical label extension task actually refer to (perceptual) properties of the items (e.g., interpreting FEP, as meaning "long item"; see Cimpian and Markman, 2005). If one of the latter explanations were true, preschoolers would have continued to rely on shape even after their knowledge about the objects had been triggered.

While our results are in accordance with Cimpian and Markman's (2005) findings, our interpretation differs from theirs. As mentioned above they attributed preschoolers' shape preference in typical word learning tasks to the fact that the experimental stimuli were simple and decontextualized; preschoolers were seen as "... incapable of identifying superordinate-level categories without perceptual support" (p. 1006). Their shape choices were thus interpreted as a reflection of the "taxonomic assumption," since shape similarity normally is a correlate of categorical relations. However, rather than taking preschoolers' shape choices as a reflection of an inability to recruit knowledge, or as a lack of knowledge, we regard them as a strategy which has been prompted by certain features of the task environment and which is applied independently of whether or not relevant categorical knowledge is available. In this sense, by providing a context and more complex objects, as Cimpian and Markman did, the salience of the shape similarity and, as a consequence, the prompting of a shape-based strategy was reduced. In fact, Cimpian and Markman found that using more "natural" items significantly reduced children's shape choices but did not increase their categorical choices. In other words, when the triggering of the shape-based strategy had been reduced children did not automatically shift to a category-based strategy. According to the "taxonomic assumption" one would have expected children's categorical choices to be dominant when the items used were more complex or contextualized and similarity between the standard and the shape match was, thus, reduced. However, the present research has shown that preschoolers' switch to the category-based strategy seems to have occurred only when prior knowledge (including categorical knowledge) was triggered and was thus easily available for their decision on how to extend a novel noun.

Our research also sheds new light on Gentner and Namy's (1999) findings (see also Namy and Gentner, 2002). As mentioned above, they found that providing two or more standards resulted in a shape-to-category shift in 4-year-olds' extensions of novel count nouns if the standards were labeled with the same novel noun, while no such shift occurred in a version with only one labeled standard. According to Gentner and Namy, the process of comparing multiple standards acts as a bridge leading from an initial perception-based category to a later, more sophisticated understanding of the category. However, while we agree that comparison processes play a major role in conceptual learning and in the development of (taxonomic) categories, Gentner and Namy's design does not allow us to clarify whether the act of comparing multiple category members actually generated new knowledge in the 4-year-olds or just triggered their conceptual knowledge about the standards, which in turn may have prompted them to switch to a category-based strategy. Our results favor the latter interpretation because we did not teach anything new to the children. All we did was to trigger preschoolers' retrieval of existing knowledge. Inviting preschoolers to compare two objects from the same superordinate category highlights their conceptual commonalities and, as a consequence, makes this knowledge easily available for their decision.

Taken together, these previous findings in combination with the present results suggest that preschoolers can use different strategies for word extensions depending on which strategy is prompted by the task environment. However, there is also a significant difference between the previous studies' findings and our own, which may be important with respect to potential explanations of the cognitive mechanisms underlying strategy choice in word extension. While some of the studies triggered specific knowledge or specific categories (e.g., Gentner and Namy, 1999), we triggered children's conceptual knowledge in a rather general way. We asked questions about all three choice objects and, in Experiment 3, we even referred, with equal intensity, to all three kinds of relations between the standard and the choice items. In other words, we triggered not only the knowledge that was relevant for a category-based noun extension. This indicates that making more information easily available supports preschoolers' shift from a single-cue strategy (e.g., shape-based strategy) to a strategy which integrates multiple (relevant) cues (e.g., category-based strategy). In other words, children's choices depend on the number of overlapping features of the items. We will elaborate this issue in more detail below.

\section{WORD EXTENSION AS A HEURISTIC DECISION STRATEGY}

As pointed out in the introduction we understand strategy as a heuristic decision-making process which selectively relies only on a subset of the information available for making an appropriate decision. Which information (and hence which strategy) is selected for decision-making depends on the information that is available (either through the task context or by retrieval from 
memory), on the predictive value of this information, and on the subject's cognitive processing abilities (e.g., Gigerenzer et al., 1999; Gigerenzer, 2008; Gigerenzer and Brighton, 2009). For example, studies with adults have shown that the amount of information that is easily available affects the amount of information that is integrated in the decision process (Bröder and Schiffer, 2003). In particular, if subjects are provided with information rather than made to retrieve it on their own, they tend to switch from a one-cue decision strategy to a strategy that integrates multiple cues.

The theoretical framework of heuristic decision-making may therefore indeed provide a suitable framework for preschoolers' label extension. It has been shown that heuristics, understood as fast and efficient decision strategies, lead to ecologically rational decisions by relying only on a subset of the total of information that is potentially relevant (e.g., Gigerenzer and Selten, 2001). Ignoring information seems to enhance the predictive value of these decision strategies for dynamic and changing environments, thus leading to robust decisions (Gigerenzer and Brighton, 2009). How, then, might this framework account for the findings of the present research?

Young children have been shown to benefit from a "single-cue strategy" for word learning (i.e., overlap in one feature): Relying on shape as a cue is a highly reliable, robust, and frugal strategy for extending words for basic-level objects that are learned early in life (e.g., Rosch et al., 1976; Smith et al., 2002; Gershkoff-Stowe and Smith, 2004). Even adults have been shown to rely on shape for doing categorization tasks when they lack knowledge about the objects concerned (e.g., Chi et al., 1981; Landau et al., 1988). At the same time, however, children learn to develop a categorybased strategy when they have to learn names for categories whose members do not resemble each other in shape (i.e., many superordinate categories). The category-based strategy can be seen as a "multiple-cue" strategy because one-cue may not be enough to identify categorical relations, particularly on a superordinate level.

In the standard label extension task, the category-defining cues are not directly provided by the task environment (i.e., they are not directly "visible") but need to be retrieved from memory. The similarity of the shape match, in contrast, is highly salient and may have prompted preschoolers to overestimate its predictive value as a cue and, consequently, may have prevented them from retrieving further information about the objects from memory. In the triggering conditions, however, more information about the objects was made easily available, and preschoolers were able to notice the larger feature overlap between the standard and the categorically related object and, as a consequence, were more likely to extend a novel label to the categorically related object. The developmental mechanisms driving the change from preschoolers to adults can thus be traced back to the availability of prior knowledge when carrying out the noun extension. Future research needs to provide the reasons for the difference in knowledge availability across the age groups. There are at least two possibilities which may not be mutually exclusive: First, preschoolers have difficulties to inhibit their reliance on the shape strategy due to premature executive functions. Thus, there should be high correlations between measures for executive functions and children's word learning strategies. Second, superordinate categories of everyday objects are highly over-learned in adults and, thus, this information is triggered automatically by the choice objects. This predicts that shape choices should increase in adults' label extensions for uncommon categories. Most importantly, future research needs to carve out the similarities and specify the differences between the theoretical approaches in order to come up with an integrative view on noun learning. For example, our approach of framing noun learning as heuristic decision-making and the attentional learning account share the underlying idea that noun learning can be described as a dynamic and flexible adaptation. However, both approaches differ with respect to how information is selected and integrated into the real time decision. Here, future studies can be designed in a way to evaluate the specific contributions of the two theories for explaining learning, selection, and integration of information within a common methodology including the same kind of stimulus material (e.g., real and artificial, solid and non-solid entities).

Taken together, we are not pitting constraint-based explanations (i.e., the taxonomic assumption) against explanations based on attentional processes (i.e., attention shifts to certain cues) to account for preschoolers' noun extensions. Rather, we try to capture preschoolers' word learning in a framework of heuristic decision strategies by establishing a link between the task structure and preschooler's individual knowledge. Preschoolers' understanding of the meaning of nouns is likely to be the result of perceptual processes (e.g., "cued attention": Smith et al., 2010) as well as knowledge at multiple levels of abstraction (e.g., Kemp et al., 2007) and will, thus, integrate different pieces of information and representations (Waxman and Gelman, 2009). Introducing heuristic decision-making (e.g., Gigerenzer et al., 1999) as a potential theoretical framework for describing preschoolers' word learning has the advantage of providing an ecologically plausible processing account with respect to which information is selected and how this information is integrated to act as a guideline for decisionmaking when novel words have to be generalized. It may thus allow us to overcome simplified dichotomies (i.e., preschoolers' reliance on either shape or taxonomic relations) and do away with the need to introduce innate constraints, an approach that has been criticized as non-informative for explaining children's word learning (see Deák, 2000). We believe that the heuristic decision-making approach has a high potential for informing about children's choices in various domains. For example, it may be particularly interesting to apply this approach to research on children's inductive reasoning in order to further resolve the discrepancy between preschoolers' label extension and property inductions with respect to categorical choices. As laid out in the introduction, we would predict that specific linguistic cues (e.g., "inside") may be responsible for prompting a category-based strategy in preschoolers' inductive inferences, whereas such cues are not provided in common label extensions tasks. Unfortunately, children's decision-making has not yet attracted much attention by the field. Therefore, our suggestions could be seen as starting points for future research. We are certain that an integrative approach in terms of decision-making theory and cognitive development research will shed new light on the understanding of children's noun extensions. 


\section{REFERENCES}

Baldwin, D. A. (1992). Clarifying the role of shape in children's taxonomic assumption. J. Exp. Child Psychol. 54, 392-416.

Booth, A. E., and Waxman, S. R. (2002). Word learning is "smart": evidence that conceptual information affects preschoolers' extension of novel words. Cognition 84, B11-B22.

Booth, A. E., Waxman, S. R., and Huang, Y. T. (2005). Conceptual information permeates word learning in infancy. Dev. Psychol. 41, 491-505.

Bowerman, M. (1978). "The acquisition of word meaning: an investigation into some current conflicts," in Development of Communication: Social and Pragmatic Factors in Language Acquisition, eds N. Waterson and C. Snow (New York: Wiley), 263-287.

Bröder, A., and Schiffer, S. (2003). Take the best versus simultaneous feature matching: probabilistic inferences from memory and effects of representational format. J. Exp. Psychol. Gen. 132, 277-293.

Bulloch, M. J., and Opfer, J. E. (2009). What makes relational reasoning smart? Revisiting the perceptualto-relational shift in the development of generalization. Dev. Sci. 12, 114-122.

Carey, S. (1985). Conceptual Change in Childhood. Cambridge, MA: Bradford Books MIT Press.

Chi, M. T. H., Feltovich, P. J., and Glaser, R. (1981). Categorization and representation of physics experts and novices. Cogn. Sci. 5, 121-152.

Cimpian, A., and Markman, E. M. (2005). The absence of a shape bias in children's word learning. Dev. Psychol. 41, 1003-1009.

Clark, E. V. (1973). "What's in a word? On the child's acquisition of semantics in his first language," in Cognitive Development and the Acquisition of Language, ed. T. E. Moore (New York: Academic Press), 65-110.

Colunga, E., and Smith, L. B. (2005). From the lexicon to expectations about kinds: a role for associative learning. Psychol. Rev. 112, 347-382.

Colunga, E., and Smith, L. B. (2008). Knowledge embedded in process: the self-organization of skilled noun learning. Dev. Sci. 11, 195-203.

Deák, G. (2000). Hunting the fox of word learning: why "constraints" fail to capture it. Dev. Rev. 20, 29-80.

Deák, G., and Bauer, P. J. (1996). The dynamics of preschoolers' categorization choices. Child Dev. 67, 740-767.

Gelman, S. A. (2003). The Essential Child. New York: Oxford University Press.

Gelman, S. A., Collman, P., and Maccoby, E. E. (1986). Inferring properties from categories versus inferring categories from properties: the case of gender. Child Dev. 57, 396-404.

Gelman, S. A., and Markman, E. M. (1986). Categories and induction in young children. Cognition 23, 183-209.

Gelman, S. A., and Markman, E. M. (1987). Young children's inductions from natural kinds: the role of categories and appearances. Child Dev. 58, 1532-1541.

Gelman, S. A., and Wellman, H. M. (1991). Insides and essences: early understandings of the non-obvious. Cognition 38, 213-244.

Gentner, D. (1978). On relational meaning: the acquisition of verb meaning. Child Dev. 49, 988-998.

Gentner, D., and Namy, L. L. (1999). Comparison in the development of categories. Cogn. Dev. 14, 487-513.

Gershkoff-Stowe, L., and Smith, L. B. (2004). Shape and the first hundred nouns. Child Dev. 75, 1098-1114.

Gigerenzer, G. (2008). Why heuristics work. Perspect. Psychol. Sci. 3, 20-29.

Gigerenzer, G., and Brighton, H. (2009). Homo heuristicus: why biased minds make better inferences. Top. Cogn. Sci. 1, 107-143.

Gigerenzer, G., and Selten, R. (2001). Bounded Rationality: The Adaptive Toolbox. Cambridge, MA: MIT Press.

Gigerenzer, G., Todd, P. M., and The ABC Research Group. (1999). Simple Heuristics That Make Us Smart. Oxford, NY: Oxford University Press.

Golinkoff, R. M., Shuff-Bailey, M., Olguin, R., and Ruan, W. (1995). Young children extend novel words at the basic level: evidence for the principle of categorical scope. Dev. Psychol. 31, 494-507.

Heyman, G. D., and Gelman, S. A. (2000a). Preschool children's use of novel predicates to make inductive inferences about people. Cogn. Dev. 15, 263-280.
Heyman, G. D., and Gelman, S. A. (2000b). Preschool children's use of trait labels to make inductive inferences. J. Exp. Child Psychol. 77, 1-19.

Imai, M., Gentner, D., and Uchida, N. (1994). Children's theories of word learning: the role of shape similarity in early acquisition. Cogn. Dev. 9, 45-75.

Imai, M., Saalbach, H., and Stern, E. (2010). Are Chinese and German children taxonomic, thematic or shape biased?: influence of classifiers and cultural contexts. Front. Psychol. 1:194. doi:10.3389/fpsyg.2010.00194

Kemler Nelson, D. G., Frankenfield, A., Morris, C., and Blair, E. (2000). Young children's use of functional information to categorize artifacts: three factors that matter. Cognition 77, 133-168.

Kemp, C., Perfors, A., and Tenenbaum, J. B. (2007). Learning overhypotheses with hierarchical Bayesian models. Dev. Sci. 10, 307-321.

Landau, B., Smith, L. B., and Jones, S. S. (1988). The importance of shape in early lexical development. Cogn. Dev. 3, 299-321.

Markman, E. M. (1989). Categorization and Naming in Children: Problems of Induction. Cambridge, MA: MIT Press.

Markman, E. M., and Hutchinson, J. E. (1984). Children's sensitivity to constraints on word meaning: taxonomic versus thematic relations. Cogn. Psychol. 16, 1-27.

Namy, L., and Gentner, D. (2002). Making a silk purse out of two sow's ears: young children's use of comparison in category learning. J. Exp. Psychol. Gen. 131, 5-15.

Rosch, E., Mervis, C. B., Gray, W. D., Johnson, D. M., and BoyesBraem, P. (1976). Basic objects in natural categories. Cogn. Psychol. 8, 382-439.

Saalbach, H., and Imai, M. (2006). "Categorization, label extension, and inductive reasoning in Chinese and German preschoolers: influence of a classifier system and universal cognitive constraints," in Proceedings of the 28th Annual Conference of the Cognitive Science Society, eds R. Sun and N. Miyake (Austin, TX: Cognitive Science Society), 703-708.
Samuelson, L. K., and Horst, J. S. (2007). Dynamic noun generalization: moment-to-moment interactions shape children's naming biases. Infancy 11, 97-110.

Smith, L. B., Colunga, E., and Yoshida, H. (2010). Knowledge as process: contextually cued attention and early word learning. Cogn. Sci. 34, 1287-1314.

Smith, L. B., Jones, S. S., and Landau, B. (1996). Naming in young children: a dumb attentional mechanism? Cognition 60, 143-171.

Smith, L. B., Jones, S. S., Landau, B., Gershkoff-Stowe, L., and Samuelson, L. K. (2002). Object name learning provides on-the-job training for attention. Psychol. Sci. 13, 13-19.

Waxman, S. R., and Gelman, R. (1986). Preschooler's use of superordinate relations in classification and language. Cogn. Dev. 1, 139-156.

Waxman, S. R., and Gelman, S. A. (2009). Early word-learning entails reference, not merely associations. Trends Cogn. Sci. 13, 258-263.

Waxman, S. R., and Kosowski, T. D. (1990). Nouns mark category relations: toddlers' and preschoolers' word learning biases. Child Dev. 61, 1461-1473.

Conflict of Interest Statement: The authors declare that the research was conducted in the absence of any commercial or financial relationships that could be construed as a potential conflict of interest.

Received: 04 August 2011; accepted: 17 October 2011; published online: 08 November 2011.

Citation: Saalbach $H$ and Schalk $L$ (2011) Preschoolers' novel noun extensions: shape in spite of knowing better. Front. Psychology 2:317. doi: 10.3389/fpsyg.2011.00317

This article was submitted to Frontiers in Developmental Psychology, a specialty of Frontiers in Psychology.

Copyright (c) 2011 Saalbach and Schalk. This is an open-access article subject to a non-exclusive license between the authors and Frontiers Media SA, which permits use, distribution and reproduction in other forums, provided the original authors and source are credited and other Frontiers conditions are complied with. 